\title{
Plant Growth-Prompting Bacteria Influenced Metabolites of Zea mays var. amylacea and Pennisetum americanum $p$. in a Species-Specific Manner
}

\author{
Faten Dhawi ${ }^{1}$, Anna Hess ${ }^{2}$ \\ ${ }^{1}$ Biotechnology Department, King Faisal University, Al-Hofuf, Al-Ahsa, Saudi Arabia \\ ${ }^{2}$ Minnesota Department of Natural Resources, Division of Ecological and Water Resources, Duluth, USA \\ Email: dr.faten.dhawi@gmail.com, falmuhanna@kfu.edu.sa, Anna.hess@state.mn.us
}

How to cite this paper: Dhaw, F. and Hess, A. (2017) Plant Growth-Prompting Bacteria Influenced Metabolites of Zea mays var. amylacea and Pennisetum americanum $p$. in a Species-Specific Manner. Advances in Biological Chemistry, 7, 161-169. https://doi.org/10.4236/abc.2017.75011

Received: August 19, 2017

Accepted: September 22, 2017

Published: September 25, 2017

Copyright $\odot 2017$ by authors and Scientific Research Publishing Inc. This work is licensed under the Creative Commons Attribution International License (CC BY 4.0).

http://creativecommons.org/licenses/by/4.0/

\begin{abstract}
Poor soil is one of the agricultural world's principal challenges, inciting the use of chemical fertilizer's to improve overall soil quality. However, the use of chemical fertilizer has significant and cascading environmental consequences. Therefore, the use of beneficial microbes' inoculation in treating poor soil is a considerably ecofriendly sustainable solution. In the current study, we supplemented nutrient-deprived soil with plant growth promoting bacteria (PGPB), Pseudomonas fluorescens. The bacterial inoculations of Pseudomonas fluorescens were added to the poor soil following two days post-sowing of Zea mays var. amylacea and Pennisetum americanum $p$. seedlings. Metabolite analyses were conducted two months after treatment for both shoots and roots using nuclear magnetic resonance method (NMR). The data indicated significant changes in 19 metabolites relative to control in both plants shoot and roots. Among these metabolites, 7 were upregulated in roots of Zea mays var. amylacea, and 9 metabolites were upregulated in roots of Pennisetum americanum $p$. The PGPB enhanced sugars (fructose, glucose, sucrose) and amino acids (glutamate, alanine and succinate) in roots, while down regulating in shoots of Pennisetum americanum $p$. The Pseudomonas fluorescens induced, predominantly, Aminoacyl-tRNA related metabolite, and Alanine, aspartate and glutamate metabolite biosynthesis in Zea mays var. amylacea), whereas PGPB induced metabolites in Pennisetum americanum $p$., dominated by up regulated carbohydrate related (starch and sucrose) metabolites. The difference in some metabolic response between the two plants indicated that PGPB influence has a species-specific manner.
\end{abstract}




\section{Keywords}

Zea mays var. amylacea, Soil, Plant Growth Promoting Bacteria,

Pseudomonas fluorescens, Pennisetum americanum p., Metabolites

\section{Introduction}

Industrialization and the associated consequences of global warming have influenced many aspects of our lives, including agricultural practices and plant production. Soil infertility is one of the most significant outcomes of global warming, due to the increased use of chemical fertilizations which are costly and harmful to environmental systems. To overcome the environmentally imbalanced systems resulting from chemical fertilizations, the use of microorganisms as biofertilizers have been explored intensively. There were many studies reporting microorganisms' ability to increase overall soil quality, including soil fertility [1] [2] and associated plant productivity, disease resistance and stress adaptation [3] [4] [5]. Plant Growth Promoting Bacteria (PGPB) have been found to increase protein expression [6], metabolites and subsequent root growth in several plants [1] [2] [7] resistance to biotic and abiotic stress [8], enriching poor nutrient soil [7]. The PGPB such as Bacillus altitudinis and Pseudomonas putida UW4 increase plant growth and subsequent biomass via producing Indole Acetic Acid (IAA) in the rhizosphere area [9] [10]. In addition, Bacillus altitudinis WR10 has been reported to increase Triticum aestivum $L$. iron tolerance [9]. Similarly, Pseudomonas sp. increased plant copper tolerance [11].

The aim of this study was to evaluate metabolic response in both shoot and roots of two plants: Zea mays var. amylacea and Pennisetum americanum, planted in poor soil (nutrient-deprived soil) when inoculated with PGPB (Pseudomonas fluorescens). Two months after inoculation, Nuclear magnetic resonance (NMR) analyses identified metabolites in both shoot and roots in both plants. The metabolic analyses indicated that PGPB induced amino acid and sugar development in root systems for both plants. The metabolic induction was associated with Aminoacyl-tRNA biosynthesis in Zea mays var. amylacea and carbohydrate pathways in Pennisetum americanum $p$.

\section{Material and Methods}

\subsection{Plant Material}

In the current study, we examined root and shoot metabolites in two types of plants, Zea mays var. amylacea and Pennisetum americanum $p$. Two-day seedling were planted in poor soil supplemented with PGPB, Pseudomonas fluorescens. The poor soil has a low water holding capacity and is poor in lime $(\mathrm{CaO})$, and nutrient such as magnesium $(\mathrm{Mg})$, nitrogen $(\mathrm{Na})$, phosphate (PO3-4), and potassium (K). The control group (C group) contained $120 \mathrm{ml}$ of $0.85 \%$ sodium chloride $(\mathrm{NaCl})$. Plant growth promoting bacteria (B group)was inoculated with 
$120 \mathrm{ml}$ of Pseudomonas fluorescens suspended in $0.85 \% \mathrm{NaCl}\left(120 \mathrm{ml} / 10^{-8}\right)$, according to a method described by Dhawi et al., [1]. Plants were watered each day in a green house at $28^{\circ} \mathrm{C}, 60 \%$ humidity and 14 hours of day light for two months.

\subsection{Metabolite Extraction}

Two months after inoculation, the experiment was terminated and shoots and roots were frozen in liquid nitrogen at $-80^{\circ} \mathrm{C}$ freezer to prepare for analyses. Metabolites extraction was modified from the Fiehn [12] method, utilizing six replicates per group. Plants samples stored in $-80^{\circ} \mathrm{C}$ were used to extract metabolites. Sample was grinded with liquid nitrogen then $0.1 \mathrm{~g}$ transferred to $2 \mathrm{ml}$ Eppendorf tube. In cold bath, $1 \mathrm{ml}$ of extraction solution (chloroform: methanol: $\mathrm{H}_{2} \mathrm{O}$ (1:2.5:1) was added to each tube. The samples were vortexed followed by addition of $60 \mathrm{ul}(2 \mathrm{mg} / \mathrm{ml})$ Ribitol (Adonitol). The samples tubes then sonicated in ice bath for $30 \mathrm{~min}$. Then centrifuged $30 \mathrm{~min}$. The supernatant transferred from each tube to a fresh vial. Samples were stored at $-80^{\circ} \mathrm{C}$ upon use for NMR.

Metabolite analyses and identification were conducted in the Minnesota Nuclear Magnetic Resonance Center MNMR (Minneapolis, MN). Samples were transferred into 1.7-mm NMR tubes and stored in the cooled SampleJet auto sampler at $\sim 6^{\circ} \mathrm{C}$ while awaiting acquisition. Each was heated to $25^{\circ} \mathrm{C}$ immediately prior to acquisition. The NMR spectra were acquired using a gradient-enhanced 1D NOESY-pre-saturation pulsesequence (noesygppr1d) for water suppression on a Bruker Avance III 700-MHz spectrometer with a TCI 1.7-mm cryoprobe. Acquisition parameters were as follows: $2 \mathrm{~s}$ pre-saturation of the water signal during the pre-scan delay, $4.1 \mathrm{~ms}$ mixing time, $2.3 \mathrm{~s}$ acquisition

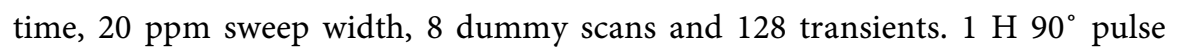
width and transmitter offset were optimized for each sample. All spectra were zero-filled to $128 \mathrm{k}$ data points, Fourier transformed with $1 \mathrm{~Hz}$ line broadening applied, and manually phased using Topspin software. Baseline correction and chemical shift referencing to the Trimethylsilylpropanoic acid (TSP) peak at 0 ppm were performed using the Processor module in Chenomx NMR Suite 8.0. The analysis identified 19 compounds (Acetate, Alanine, Choline, Citrate, Formate, Fructose, Gallate, Gluconate, Glucose, Glutamate, Glutamine, Isoleucine, Malate, Succinate, Sucrose, Threonine, Tyrosine, Valine and trans-Aconitate) quantified relative to the $0.15 \mathrm{mM}$ TSP. Theconcentration was reported by the requester using the Profiler module in Chenomx NMR Suite 8.1 with theChenomx 700-MHz compound Library.

\section{Statistical Analyses}

The metabolite data were normalized and subjected to multivariate analyses with Partial Least Squares-Discriminant Analysis (PLS-DA) using Metabo Analyst [13]. The PLS-DA analyses identified metabolite variation relative to the control. Metabolites that expressed significant differences relative to control were sub- 
jected to an integrating enrichment analyses and pathway topology using KEGG database (http://www.genome.jp/kegg/pathway.html) to determine their rolein plant development [13].

\section{Results and Discussion}

Metabolite responses to various conditions are the markers of a biological system's ability to cope with different effectors. The identification of metabolites enhances our insight of biological system interactions with environment; thus, they can be utilized to gain improved plant development results using metabolic engineering. Metabolite analyses identified 19 compounds that were affected relative to the control (Acetate, Alanine, Choline, Citrate, Formate, Fructose, Gallate, Gluconate, Glucose, Glutamate, Glutamine, Isoleucine, Malate, Succinate, Sucrose, Threonine, Tyrosine, Valine and Trans-Aconitate) in both plants (Zea mays var. amylacea and Pennisetum americanum p.) shoots and roots (Figure 1 and Figure 2(a), Figure 2(b)). Shoot B group in Zea mays var. amylacea indicated upregulation in five metabolites (Alanine, Glutamate, Valine, Isoleucine and sucrose) (Figure 1(a)). Whereas, shoot B group in Pennisetum americanum $p$. indicated up regulation in seven metabolites (Sucrose, Glucose, Fructose, Gallate, Threonine, Tyrosine and trans-Aconitate) (Figure 2(a)).

Root B group in Zea mays var. amylacea indicated up regulation in 9 metabolites (Alanine, Choline, Fructose, Gallate, Glutamate, Glutamine, Succinate, Sucrose and Threonine).Conversely, Root B group in Pennisetum americanum $p$. indicated up regulation in 12 metabolites (Alanine, Choline, Citrate, Fructose, Gallate, Glucose, Glutamate, Isoleucine, Malate, Threonine, Tyrosine and Valine) (Figure 1(b) and Figure 2(b)). There was no overlap or interaction between metabolite response in control and B group in either plants (Zea mays var. amylacea and Pennisetum americanum p.) (Figure 1 and Figure 2(c), Figure 2(d)).

The association of plant growth promoting bacteria was reported to enhance poor soil (nutrient deprived) element availability, therefore enhancing plant productivity [1] [2] and plant stress tolerance [1] [2] [4] [5]. In addition, plant stress tolerance and progress impacted by PGPB associated with metabolic and protein induction shift to serve plant growth and aid overall increase in biomass [1] [2] [6]. In our study, the PGPB induced nine metabolites in Zea mays var. amylacea root, five of them (Glutamine, Valine, Alanine, Threonine and Tyrosine) associated with Aminoacyl-tRNA biosynthesis and three of them (Alanine, Glutamine and Succinic acid) associated with Alanine, aspartate and glutamate metabolism. The Aminoacy l-tRNA biosynthesis ensure efficient protein translation [14]. While Alanine, aspartate and glutamate metabolism is essential for signaling and nitrogen source [15]. Similarly, the upregulated metabolites in Zea mays var. amylacea shoot (Valine, Alanine and Isoleucine) have similar role to Aminoacy l-tRNA biosynthesis, confirming the importance of PGPB in enhancing amino acidsand nitrogen availability in nutrient deprived soil [16] [17]. 


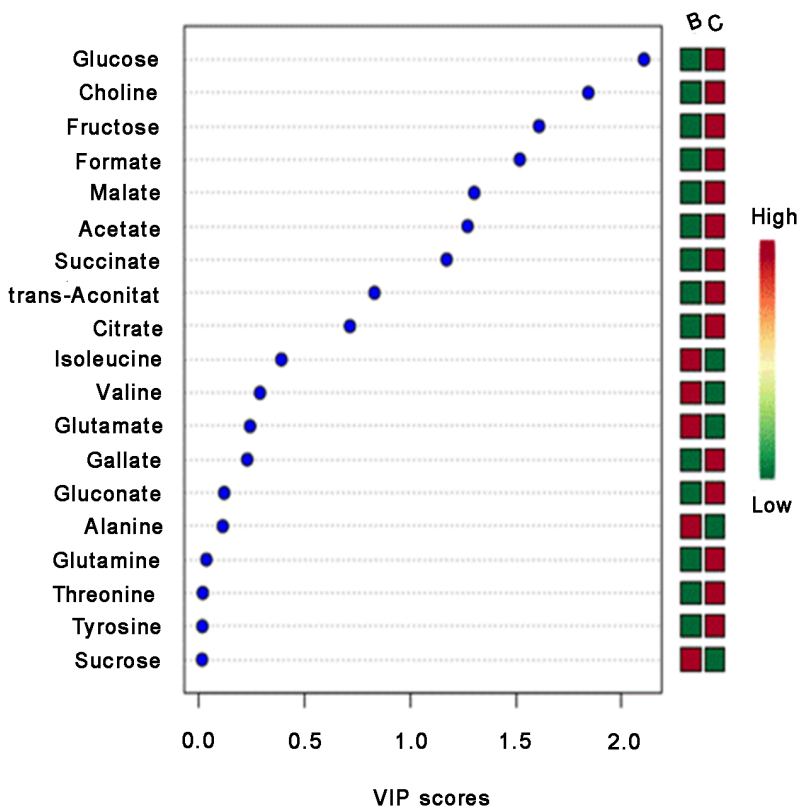

(a)

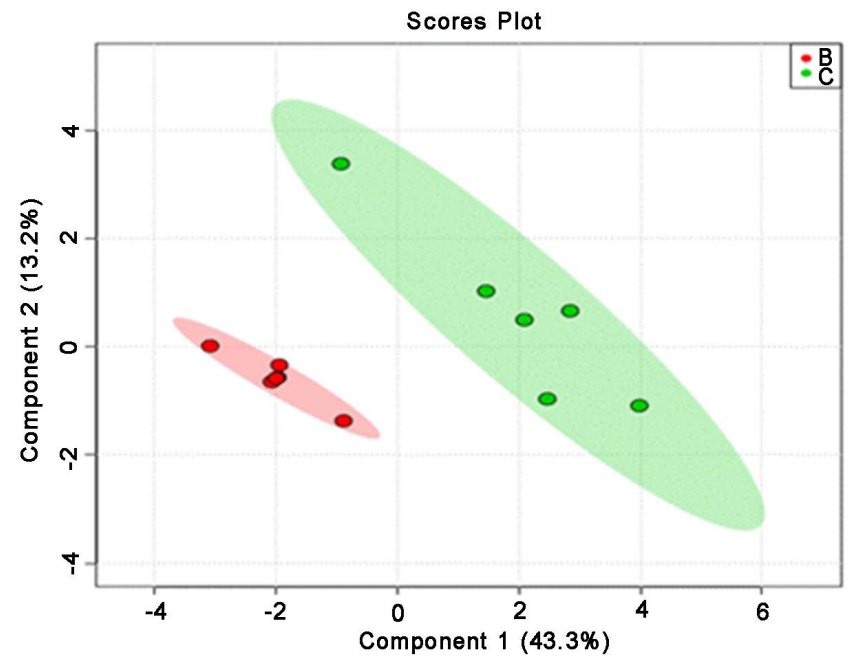

(c)

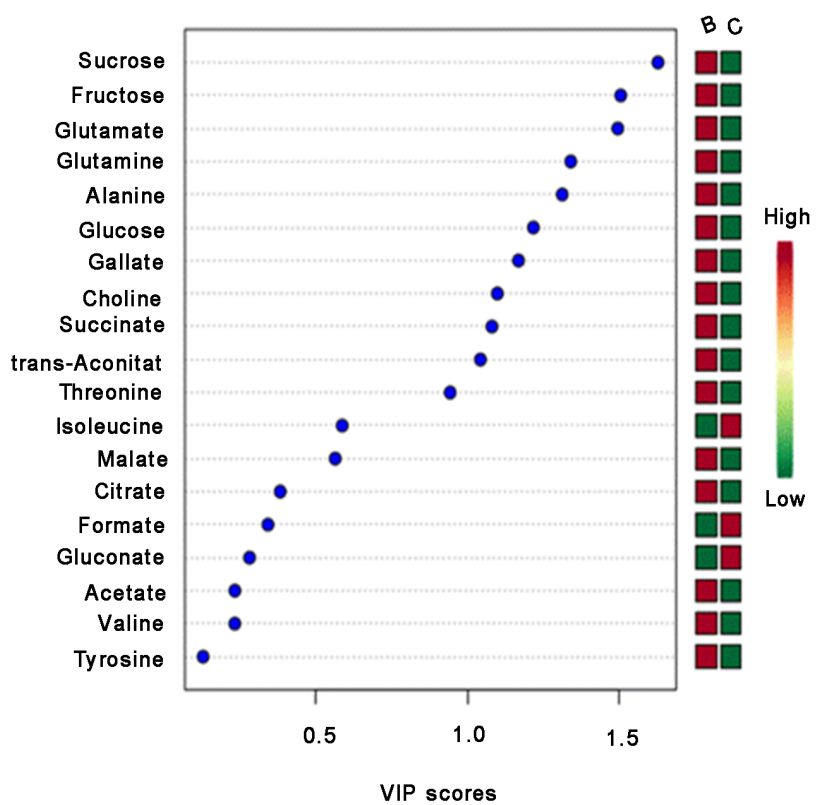

(b)

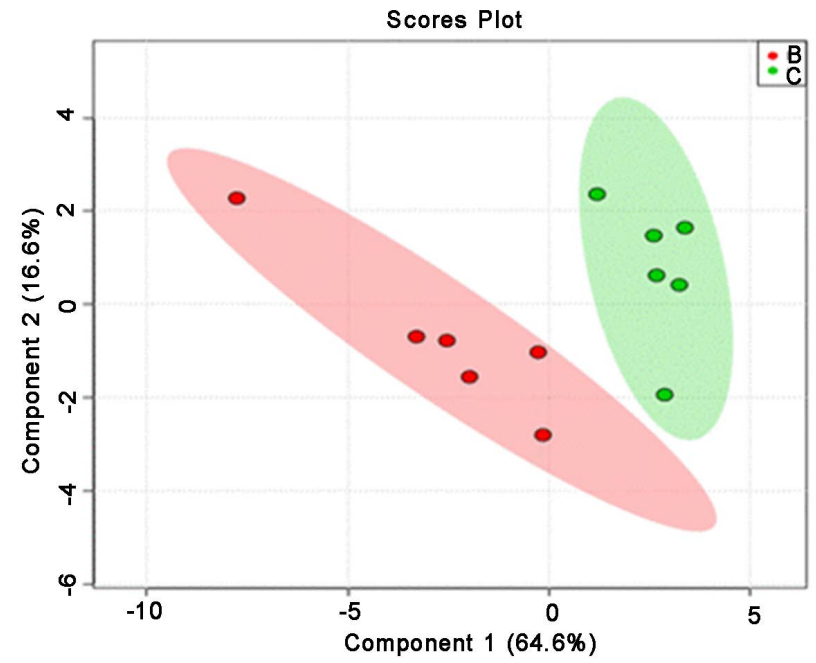

(d)

Figure 1. Metabolite response to different microbial inoculations in Zea mays var. amylacea, (a) shoot metabolites (b) root metabolites, both (a) and (b) represent the PLS-DA measurements. The score of variable importance in projection (VIP) on the left and the colored boxes on the right indicate the relative concentrations of the corresponding metabolite in each group under study. The metabolites interactions are relative to control (c) shoot and (d) root metabolites. Different letters represent groups as B: plant growth promoting bacteria and C: control group, displayed in (c) and (d).

In Pennisetum americanum p., PGPB induced 12 root metabolites; four (Alanine, Isoleucine, Threonine and Tyrosine) metabolites involved in Aminoacyl-tRNA biosynthesis, two (fructose and glucose) associated with Starch and sucrose, and two metabolites (Threonine and Isoleucine) associated with Valine, leucine and isoleucine biosynthesis. Additionally, Pennisetum americanum $p$. shoot induced metabolites (Fructose, Sucrose and Glucose) associated with Starch and sucrose metabolism and two metabolites (Sucrose and Glucose) 


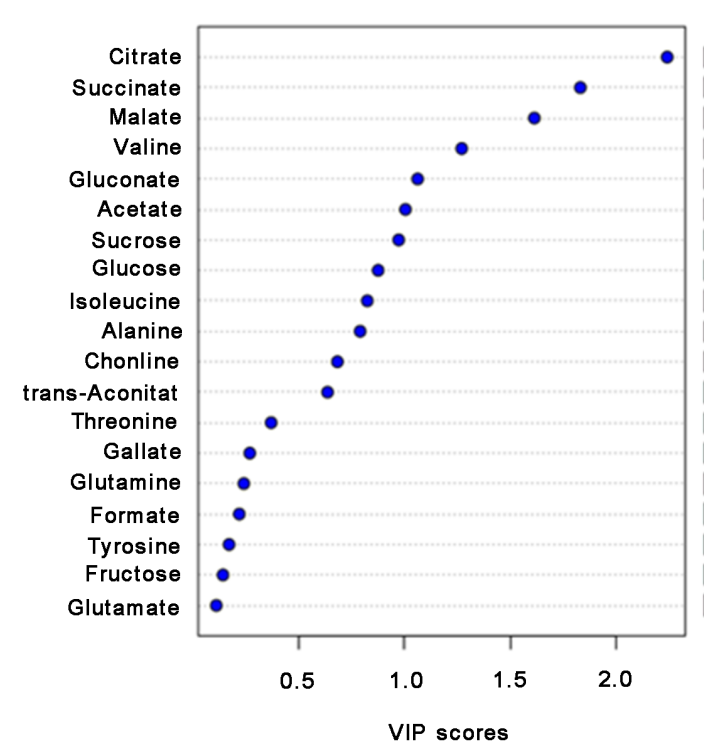

(a)

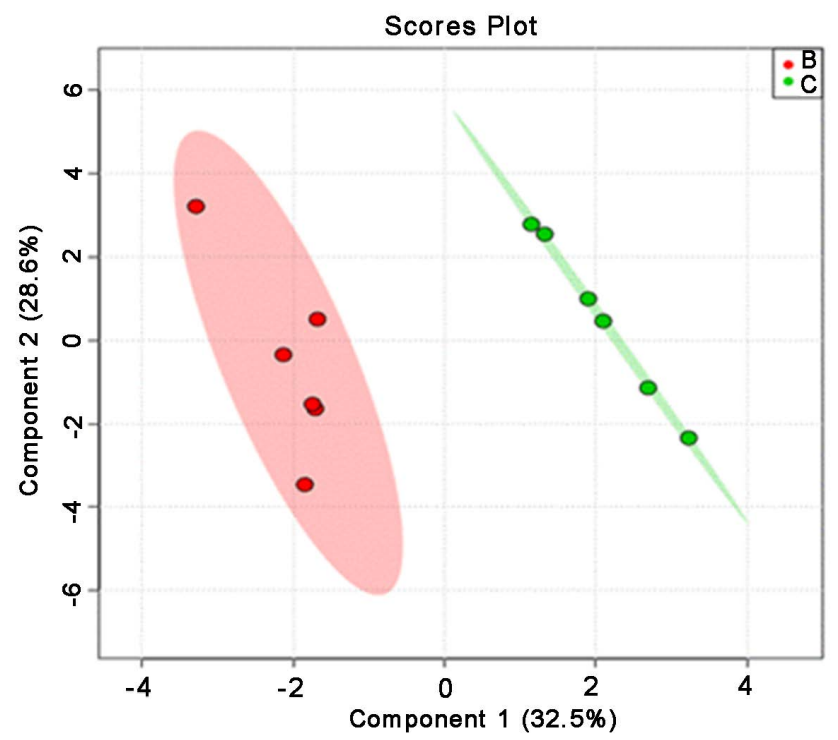

(c)

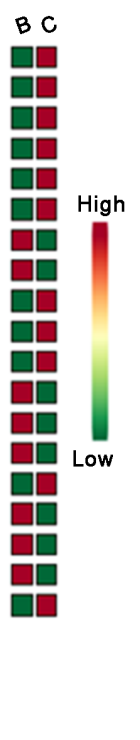

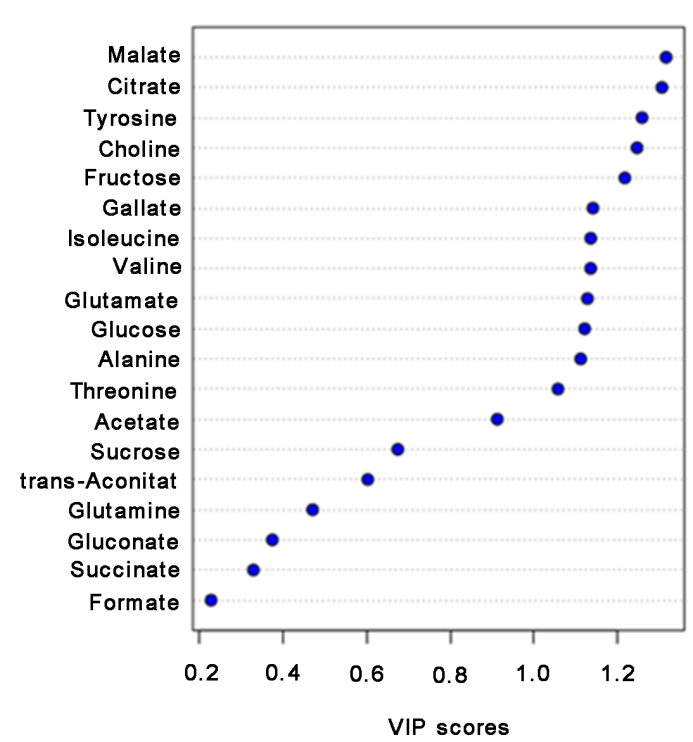

(b)

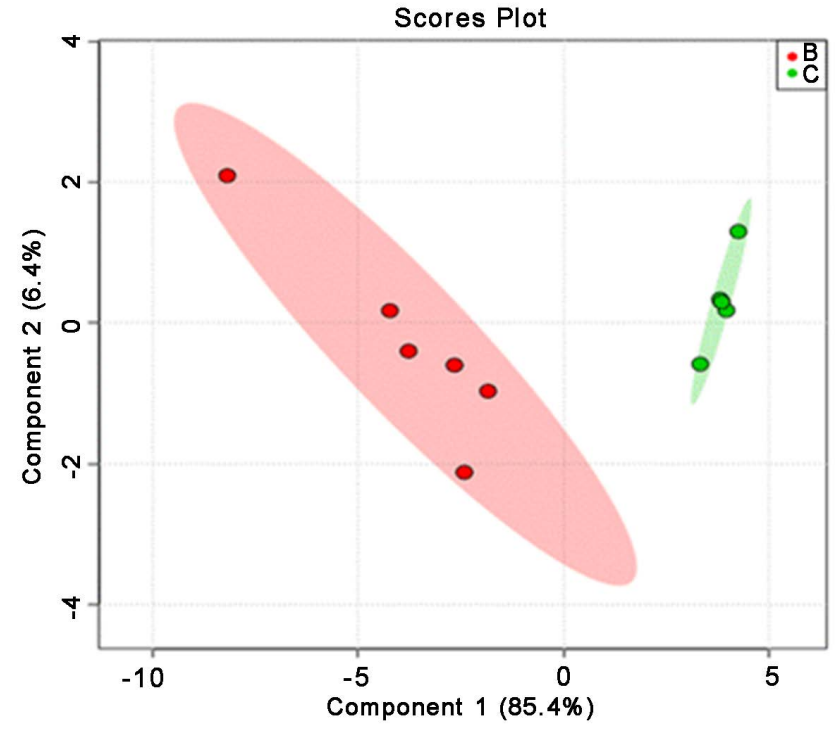

(d)

Figure 2. Metabolite response to different microbial inoculations in Pennisetum americanum, (a) shoot metabolites (b) root metabolites, (a) and (b) represent the PLS-DA measurements. The score of variable importance in projection (VIP) on the left and the colored boxes on the right indicate the relative concentrations of the corresponding metabolite in each group under study.The metabolites interactions are relative to control (c) shoot and (d) root metabolites. Different letters represent groups as B: plant growth promoting bacteria and C: control group.

associated with Galactose metabolism. The current study results differ from earlier studies that indicated PGPB induced metabolites related to fatty acids, glyoxylate and dicarboxylate metabolism in sorghum and maize [1] [2].

The PGPB generated different roles in each plant species' metabolic pathways, where the Starch and sucrose metabolism dominated up regulated metabolites in Pennisetum americanum $p$, and the metabolites related to Aminoacyl-tRNA biosynthesis were up regulated in Zea mays var. amylacea. The common me- 
tabolites between the two plants root system were five (Alanine, Choline, Fructose, Gallate and Glutamate). These metabolites have critical roles in plant physiology and participate in major pathways. One of the major roles of PGPB is to increase plant ability to tolerate stress, represented in the current study by poor (nutrient deprived) soil. Metabolites such as Choline are involved in Glycerophospholipid metabolism and subsequently increase plants stress tolerance [18]. Alanine is involved in Selenoamino acid metabolism; Alanine, aspartate and glutamate metabolism, Aminoacyl-tRNA biosynthesis and Carbon fixation in photosynthesis. In addition, sugars such as Fructose are involved in Amino sugar and nucleotide sugar metabolism and Starch and sucrose metabolism. The common upregulated metabolites impacted by PGPB were involved in amino acid and carbohydrate synthesis, which was reflected on the two plants (Zea mays var. amylacea and Pennisetum americanum p.) growth and tolerance in poor elements soil condition. This is equivalent to an earlier study that indicated the ability of PGPB to increase carbohydrate metabolism and amino acid metabolism and related proteins, to subsequently promote growthand increase plant detoxification [6] [19].

A total of 19 metabolites were identified as being impacted by PGPB of Pseudomonas fluorescens innutrient deprived soil using NMR based analyses. The analyses identified significant upregulation in comparison to control in both plants root system while shoot indicated minor changes. The 12 upregulated metabolites in Pennisetum americanum p. root and the 9 upregulated metabolites in root of Zea mays var. amylacea serve a critical role in stress tolerance and carbohydrate and amino acid synthesis. The PGPB inoculation using Pseudomonas fluorescens indicated a positive influence on both plants (Zea mays var. amylacea and Pennisetum americanum p.) growth and stress resistance. However, the difference in some metabolic response indicated that PGPB influence has species-specific manner.

\section{Conclusion}

Overall, this study strongly suggests that the use of PGPB Pseudomonas fluorescens can improve soil quality (nutrient condition) without the use of commercial artificial chemical fertilizers.

\section{Acknowledgements}

We would like to thank Minnesota Nuclear Magnetic Resonance Center (420 Delaware, Minneapolis, MN (http://nmr.umn.edu/about-mnmr) for the help in interpretation of metabolites results.

\section{References}

[1] Dhawi, F., Datta, R. and Ramakrishna, W. (2015) Mycorrhiza and PGPB Modulate Maize Biomass, Nutrient Uptake and Metabolic Pathways in Maize Grown in Mining-Impacted Soil. Plant Physiology and Biochemistry, 97, 390-399. 
https://doi.org/10.1016/j.plaphy.2015.10.028

[2] Dhawi, F., Datta, R. and Ramakrishna, W. (2016) Mycorrhiza and Heavy Metal Resistant Bacteria Enhance Growth, Nutrient Uptake and Alter Metabolic Profile of Sorghum Grown in Marginal Soil. Chemosphere, 157, 33-41.

https://doi.org/10.1016/j.chemosphere.2016.04.112

[3] Dimkpa, C., Weinand, T. and Asch, F. (2009) Plant-Rhizobacteria Interactions Alleviate Abiotic Stress Conditions. Plant, Cell \& Environment, 32, 1682-1694. https://doi.org/10.1111/j.1365-3040.2009.02028.x

[4] Grover, M., Ali, S.Z., Sandhya, V., Rasul, A. and Venkateswarlu, B. (2011) Role of Microorganisms in Adaptation of Agriculture Crops to Abiotic Stresses. World Journal of Microbiology and Biotechnology, 27, 1231-1240. https://doi.org/10.1007/s11274-010-0572-7

[5] Glick, B.R. (2012) Plant Growth-Promoting Bacteria: Mechanisms and Applications. Scientifica, 2012.

[6] Dhawi, F., Datta, R. and Ramakrishna, W. (2017) Proteomics Provides Insights into Biological Pathways Altered by Plant Growth Promoting Bacteria and Arbuscular Mycorrhiza in Sorghum Grown in Marginal Soil. Biochimica et Biophysica Acta (BBA)-Proteins and Proteomics, 1865, 243-251.

https://doi.org/10.1016/j.bbapap.2016.11.015

[7] Dhawi, F. and Hess, A. (2017) Poor-Soil Rhizosphere Enriched with Different Microbial Activities Influence the Availability of Base Elements. Open Journal of Ecology, 7, 495. https://doi.org/10.4236/oje.2017.78033

[8] Choudhary, D.K., Kasotia, A., Jain, S., Vaishnav, A., Kumari, S., Sharma, K.P. and Varma, A. (2016) Bacterial-Mediated Tolerance and Resistance to Plants under Abiotic and Biotic Stresses. Journal of Plant Growth Regulation, 35, 276-300. https://doi.org/10.1007/s00344-015-9521-x

[9] Sun, Z., Liu, K., Zhang, J., Zhang, Y., Xu, K., Yu, D., et al. (2017) IAA Producing Bacillus altitudinis Alleviates Iron Stress in Triticum aestivum L. Seedling by Both Bioleaching of Iron and Up-Regulation of Genes Encoding Ferritins. Plant and Soil, $1-11$.

[10] Tabatabaei, S., Ehsanzadeh, P., Etesami, H., Alikhani, H.A. and Glick, B.R. (2016) Indole-3-Acetic acid (IAA) Producing Pseudomonas Isolates Inhibit Seed Germination and a-Amylase Activity in Durum Wheat (Triticum turgidum L.). Spanish Journal of Agricultural Research, 14, 0802.

[11] Rajkumar, M., Bruno, L.B. and Banu, J.R. (2017) Alleviation of Environmental Stress in Plants: The Role of Beneficial Pseudomonas spp. Critical Reviews in Environmental Science and Technology, 1-36. https://doi.org/10.1080/10643389.2017.1318619

[12] Fiehn, O. (2006) Metabolite Profiling in Arabidopsis. Methods in Molecular Biology, 323, 439-447. https://doi.org/10.1385/1-59745-003-0:439

[13] Xia, J. and Wishart, D.S. (2016) Using Metaboanalyst 3.0 for Comprehensive Metabolomics Data Analysis. Current Protocols in Bioinformatics, 55, 1-91.

[14] Moore, M., Gossmann, N. and Dietz, K.J. (2016) Redox Regulation of Cytosolic Translation in Plants. Trends in Plant Science, 21, 388-397.

[15] Forde, B.G. and Lea, P.J. (2007) Glutamate in Plants: Metabolism, Regulation, and Signalling. Journal of Experimental Botany, 58, 2339-2358. https://doi.org/10.1093/jxb/erm121

[16] Przemieniecki, W.S., Kurowski, P.T. and Karwowska, A. (2015) Plant Growth Pro- 
moting Potential of Pseudomonas sp. SP0113 Isolated from Potable Water from a Closed Water Well. Archives of Biological Sciences, 67, 663-673. https://doi.org/10.2298/ABS141002029P

[17] Kraiser, T., Gras, D.E., Gutiérrez, A.G., González, B. and Gutiérrez, R.A. (2011) A Holistic View of Nitrogen Acquisition in Plants. Journal of Experimental Botany, 62, 1455-1466. https://doi.org/10.1093/jxb/erq425

[18] Vurukonda, S.S.K.P., Vardharajula, S., Shrivastava, M. and SkZ, A. (2016) Enhancement of Drought Stress Tolerance in Crops by Plant Growth Promoting Rhizobacteria. Microbiological Research, 184, 13-24.

[19] Du, N., Shi, L., Yuan, Y., Li, B., Shu, S., Sun, J. and Guo, S. (2016) Proteomic Analysis Reveals the Positive Roles of the Plant-Growth-Promoting Rhizobacterium NSY50 in the Response of Cucumber Roots to Fusarium Oxysporum f. sp. Cucumerinum Inoculation. Frontiers in Plant Science, 7, 1859.

https://doi.org/10.3389/fpls.2016.01859 